\title{
EFFICIENT ENERGY UTILIZATION IN WIRELESS SENSOR NETWORKS: AN ALGORITHM
}

M. N. Khan

Department of Electrical Engineering, University of Lahore, Lahore, (Pakistan)

*E-mail: muhammad.nasir@ee.uol.edu.pk

S. O. Gilani

SMME, National University of Sciences and Technology, Islamabad, (Pakistan)

M. Jamil

SMME, National University of Sciences and Technology, Islamabad, (Pakistan)

A. Shahzad

Department of Mechanical Engineering, University of Lahore, Lahore, (Pakistan)

A. Raza

Department of Electrical Engineering, University of Lahore, Lahore, (Pakistan) 


\section{ABSTRACT}

In the era of latest technologies, wireless sensor network (WSN) is becoming more popular in many applications: e-health, e-commerce, banking, farming and many others. However, WSNs have limitations in the sense of processing power, life time and data gathering. Among the above said issues, energy efficiency is the main hindrance of WSN deployment. Different data gathering schemes such as low energy adaptive clustering hierarchy (LEACH) and power efficient gathering in sensor information systems (PEGASIS) have been proposed. However, LEACH and PEGASIS do not provide optimal results for the energy consumption problem and are not feasible to implement. In this research paper, energy efficiency in terms of data gathering in WSN is presented. In this research work, a combined flavor of particle swarm optimization (PSO) with simulated annealing (SA) is given. A novel algorithm is proposed in which best chain formation procedure is adopted. Using the proposed algorithm, a balance energy utilization occurred between nodes, which results in increasing the network performance. Simulation results are obtained and compared with other schemes, which shows better performance as compared to LEACH and PEGASIS.

\section{KEYWORDS}

Wireless sensor network (WSN), low energy adaptive clustering hierarchy (LEACH), power efficient gathering in sensor information systems (PEGASIS), base station (BS), sensor nodes $(\mathrm{SN})$.

\section{INTRODUCTION}

In the recent era, wireless sensors have become most prominent subsystem in many applications: e-Health, e-commerce, e-banking, farming, habitat monitoring, forest fire detection [1,2]. Wireless sensor networks (WSNs) are composed of one to many sensor nodes in a sensing area. Sensor nodes (SNs) are deployed in large area comprising of a base station (BS), which is located at variable distances. The SNs locating at larger distance from the BS, consume more energy while communicating or transferring information to the BS. The nodes locating at near distance dissipate less energy while others dissipate more energy. The energy dissipation depends on distance and communication time during data gathering $[3,4]$. Thus energy efficiency in data gathering is a major task to count in WSN design.

In a WSN small SNs are known to be the basic components on which processing is done. SNs are smaller in size, require low power, less memory and least expensive and can communicate over short distance $[1,5]$. Figure 1 is an example of a WSN, where a large number of SNs (spread in the sensing area) combine to make a fully operational sensor network. The SNs collect data and then forward it to the BS through a leader node (i.e., cluster head $(\mathrm{CH})$ ). The crucial task of SN is to listen an event and respond quickly by sending information to the sink node or BS [5]. 


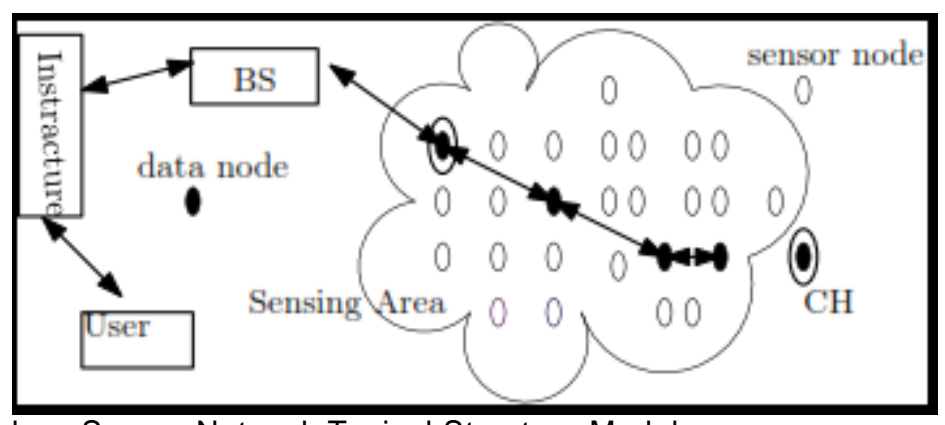

Figure 1. Wireless Sensor Network Typical Structure Model.

SNs in a WSN have very limited energy, it is hard to recharge remotely or change their batteries more often. To cope with this issue, we need to have an energyefficient data gathering protocol for balancing the energy dissipation among SNs of the network. For WSN to be efficient and acceptable, following requirements are very crucial [6-10];

The network lifetime enhancement.

Energy efficient deployment schemes.

Need for an energy-efficient data gathering protocol.

Recently, various data gathering schemes including the low energy adaptive clustering hierarchy (LEACH) [11] and power efficient gathering in sensor information systems (PEGASIS) [12] have been suggested. The LEACH protocol provides solutions for energy utilization problem. In LEACH, SNs with higher energy are chosen as a $\mathrm{CH}$ randomly and $\mathrm{CH}$ communicates to the $\mathrm{BS}$. The $\mathrm{CH}$ forwards data to the $\mathrm{BS}$ also causes energy dissipation which is not required. The un-necessary dissipation limits the applicability of LEACH for WSNs. On the other hand, PEGASIS protocol is proposed for further improvement in LEACH protocol. However, in PEGASIS, greedy chain formation allows all nodes to become the leader at the same time, which needs more rounds in forwarding data to the BS. This way of energy utilization is a big hurdle in PEGASIS implementation.

Both algorithms do not provide optimal results in terms of energy efficiency problem. We present particle swarm optimization (PSO) with simulated annealing (SA) for efficient chain formation and ensures minimum energy consumption. For the selection of efficient leader that will communicate to the sink, data gathering chain for done with SA-PSO. In a chain a leader is selected based on its residual energy $E_{\text {resid }}$ and each node can transmit information to its neighboring node, this result in increased network performance as balance energy utilization occurred between nodes. Following are the major contribution of the proposed research work;

A new scheme for efficient energy is developed and tested as compared to other protocols using extensive simulations. It is shown from simulation results that the proposed protocol can prolong the network lifetime.

The SA-PSO maximizes the performance in terms of energy efficiency and also increases the network lifetime.

In our scheme all the nodes contribute in communication to the BS and thus it eliminates unequal energy consumption of individual nodes.

The simulation results also show significant improvements as compared to LEACH and PEGASIS. Our further goal is to compare the SA-PSO performances with ant colony optimization (ACO) technique. 


\section{PRE-LIMINARIES}

Different data gathering schemes and heuristic algorithms have been suggested in [1-4]. Following are the main ones;

Conventional PSO and its variants.

LEACH and its variants

PEGASIS

Before discussing about the LEACH and PEGASIS, brief description of PSO and its variant are considered to be compulsory, because the proposed algorithm is the combination of PSO and SA.

Basically LEACH is cluster based routing protocol, consists of distributed clusters. In LEACH protocol, $\mathrm{CH}$ is randomly selected and this is constantly rotated to distribute energy load along all node in the entire network. In LEACH protocol, to decrease the load of transmitted data, the $\mathrm{CH}$ node compress data arriving from the sensor nodes and send an aggregated packet of data to the BS [7]. The drawback of LEACH protocol is that it randomizes he selection of cluster heads for equal energy dissipation. Whereas the PEGASIS protocol uses a greedy chain to sink. To overcome these drawbacks, we use particle swarm optimization with simulated annealing (SA).

\subsection{Conventional PSO}

PSO is a swarm intelligence based optimization technique. PSO was first developed in [13], inspiring from the social behavior of flocking birds. A wide variety of particles are exploited to constitute a swarm and it moves in various direction of sensing area for locating the best possible solution. Each $\mathrm{SN}$ in the sensing area keeps tracking the coordinates of optimum solution or fitness and is known as the pbest. There is also another value called the gbest that is obtained the particle in the neighborhood. The position $Y$ of each particle $m$ in the given space $D$ is formulate as $[13,14]$,

$Y_{i}=\left[y_{m_{1}}, y_{m_{2}}, \ldots, y_{m_{D}}\right]$.

The previous best position stored by each particle as pbest,

$P_{i}=\left[p_{m_{1}}, p_{m_{2}}, \ldots, p_{m_{D}}\right]$,

and the velocity for each dimension is given by,

$V_{i}=\left[v_{m_{1}}, v_{m_{2}}, \ldots, v_{m_{D}}\right]$.

The position and velocity updates are given respectively as,

$y_{m}=\left[m_{d}+v_{m_{d}}\right]$

$v_{m_{d}}=\left[v_{m_{d}}+s_{1} q_{1}\left(\right.\right.$ pbest $\left.-y_{m_{d}}\right)+s_{1} q_{1}\left(\right.$ gbest $\left.\left.-y_{m_{d}}\right)\right]$,

where $d$ is the dimension, $s_{1}$ and $s_{2}$ denote positive constants, , $q_{1}$ and $q_{2}$ are random numbers between [0-1], $y_{m}$ is position of a particle, $v_{m}$ is velocity of a particle. PSO becomes popular because of its superior performance in may application and was used with combination of other techniques as a hybrid algorithm $[15,16]$. On the other hand, SA proposed in [17] is found to be most extensive optimization technique. It is stochastic process based on Metropolis Law [8] that search for the best optimal solution. It discusses about two factors: - the energy factor and temperature factor $\theta$, where $\theta$ is referred to as annealing temperature. The value of $\theta$ is decreased by the cooling schedule. The region of best point is attained as $\theta$ approaches the lower-limit [1]. 


\subsection{Leach and its variants}

LEACH is a hierarchical routing protocol [11,15] and is a self-organizing scheme. In LEACH, SNs are divided into multiple clusters. Each cluster calculates its $\mathrm{CH}$, which collects data from all neighboring nodes in the cluster. Then $\mathrm{CH}$ forwards the aggregated data to the BS. In this process, $\mathrm{CH}$ consumes more energy if it falls near to the BS, which results its quick death [15]. To overcome this problem, LEACH randomizes the selection of $\mathrm{CH}$, in order to balance the energy dissipation. LEACH can be found in many application $[11,15,18]$.

In LEACH, once the cluster is formed, all the nodes decide to be the $\mathrm{CH}$ with probability $p_{r}$ and broadcast its advertisement to all neighbors. A non-cluster head node finds its cluster by selecting the $\mathrm{CH}$, which has the least energy. To balance the load, the $\mathrm{CH}$ data message is delivered in a sequence of node. When a $\mathrm{CH}$ dies, the cluster is not operated. It is assumed that $\mathrm{CH}$ has long transmission range so that network can have long lifetime. But it is not a good assumption in terms of signal propagation. Although LEACH was found to be useful, but it still has problem of short network lifetime because of inefficiently consumed energy $[15,19]$. To overcome issues various variants of LEACH, i.e., LEACH-C, Multi-hop LEACH, Energy-LEACH, MOD-LEACH and many more were suggested in $[11,20,21,22]$.

\subsection{Pegasis and its variants}

PEGASIS is an improved form also referred to as an extension of LEACH protocol. PEGASIS greedy chain formation based algorithm having chain construction and fusion functions [12]. In PEGASIS, each SN has global information of its whole sensing network and full knowledge of location of its neighboring nodes. The chain formation is initialized by a node over the furthest distance from BS. After the connection of node in chain, all its correspondence turned off from remaining chain formation process.

It is assumed that $\mathrm{SNs}$ are capable to vary the signal strength after getting the global information. By doing so, energy consumption can be minimized up to an extent that the network lifetime is maximized. It is presented that PEGASIS is comparatively more energy efficient as compared to LEACH in the sense of network lifetime [20]. The energy is saved by forwarding the aggregated data rather than bulk data to BS. The drawback of PEGASIS is that due to greedy chain formation process, whole data transmission to BS takes more time, which causes higher latency. PEGASIS have been modified and proposed by many researcher [22-27].

\section{PROPOSED DATA GATHERING SCHEME}

In the proposed energy efficient scheme, a chain is supposed to be formed allowing the transmission of data by individual nodes. This transmission of data is carried out to the BS for unequal number of times. To achieve our design objective, we use PSO [13] and SA [17] that result in energy efficient network by the individual nodes and enhanced network performance.

\subsection{Selection of leader}

An optimal chain formation using SA is presented and the selection of a leader (i.e., $\mathrm{CH}$ ) is done for communication. In our scheme there is data transmission 
between the closed neighbors and the node become the leader in their turn depending on its residual energy and location. In the proposed scheme a chain is formed such that all nodes have equal rights to become the leader. The network lifetime is maximized because of transmitting unequal number of times by individual node to the BS based on its $E_{\text {resid }}$ and thus it eliminates unequal energy consumption of individual nodes, which results in best performance than LEACH and PEGASIS.

Once a sub-optimal chain formation is done for the first time, a node with maximum value of $\frac{E_{\text {resid }}}{D^{\gamma}}$, here $D$ is the distance of the BS from that node, is to be located (i.e., search a node with maximum energy) before initializing the round of data gathering. In this process, a node, which gives the maximum value of $\frac{E_{\text {resid }}}{D}$ is chosen as the leader. The multi-path fading (i.e., distance power loss) channel mode is assumed in case the leader is concerned to communicate with the distant BS.

\subsection{Data gathering algorithm: SA-PSO}

To overcome drawbacks of LEACH and PEGASIS, we present a scheme in which an efficient leader is chosen for communicating to BS. The SA algorithm is combined with PSO to efficiently solve the chain formation problem. Our aim is to minimize the energy usage by $\mathrm{SNs}$ in forming an optimal chain over which the data gathering is done. To simplify the model, we consider the same radio model as is presented in [7].

To eliminate the local minima trapping of the existing algorithm, the proposed method of application of SA with PSO solve two major issues: increase the diversity of the particle and efficiently solve chain formation problem. Let's consider $\$ \mathrm{n} \$$ total nodes and solution space $U$ which is $U$ $\varepsilon\{1,2,3, \ldots, \mathrm{n}\}$ a collection of arrangement and the consecutive nodes are linked together by a direct link. The combination, i.e., $C$ denotes a chain and $C=U_{i} \mid C$ represents a permutation of $(1,2, \ldots \mathrm{n})$.

\section{Proposed algorithm}

\section{Step 1: Initilization.}

Initializing all parameters $\left(\alpha, \theta_{\mathrm{i}}, \theta_{\mathrm{f}}\right)$, where $0.7 \leq \alpha \leq 1$.

The randomly selected swarm of $m$ particles is expressed as $C_{1}, C_{2}, C \ldots C_{m}$

\section{Step 2: Locating a local best chain.}

Search for the local best chain for a given $\theta_{\mathrm{i}}$, based on binary swapping resulting $\mathrm{C}_{\text {ilbest }}$ is find out with the help of binary swapping between the two positions of $\mathrm{G}_{\text {old }} \cdot \mathrm{C}_{\text {old }}$, updated by the newly formed chain.

$\mathrm{C}_{\text {old }}=\mathrm{C}_{\text {new }}=\mathrm{C}_{\text {ipbest }}=$ Local best solution of particle

Step 3: Updating gbest values.

The newly formed chain is updated by the following rule:

$$
\begin{aligned}
\mathrm{C}_{\text {ipbest }}=\mathrm{C}_{\text {ilbest }} \text { if } & \left\{\mathrm{f}\left(\mathrm{C}_{\text {ilbest }}\right)-\mathrm{f}\left(\mathrm{C}_{\text {ipbest }}\right)\right\}<0 \\
= & \mathrm{C}_{\text {ipbest }} \text { if }\left\{\mathrm{f}\left(\mathrm{C}_{\text {ilbest }}\right)-\mathrm{f}\left(C_{\text {ipbest }}\right)\right\} \geq 0
\end{aligned}
$$

Comparing the $\mathrm{C}_{\text {ipbest }}$ values

$$
\mathbf{C}_{\text {gbest }}=\min \left\{\mathbf{f}\left(\mathbf{C}_{\text {ipbest }}\right)\right\}
$$




\section{Step 4: Formation of new chain.}

A new chain $\mathrm{C}_{\text {gbest }}$ is formed from the $\mathrm{C}_{\text {ipbest }}$ by crossing method.

\section{Step 5: Loop.}

The algorithm comes to end if the value of temperature $\theta(t)$ is less than or equal to $\theta_{\mathrm{f}}$. The best chain formed is $C_{\text {gbest }}$,else move to step 2 .

\section{SYSTEM DISSIPATION MODEL}

Radio model also referred to the energy dissipation model is the most common model known in WSNs. Block diagram of the energy dissipation model is shown in Fig. 2. During the processing of different tasks, SNs dissipate energy while transmission and reception of data from/to BS. Let's assume that SNs consume the transmitted energy $E_{T x}$ in transmitting a $k$-bit packet over a distance $d$ meters as shown in Fig. 2 .

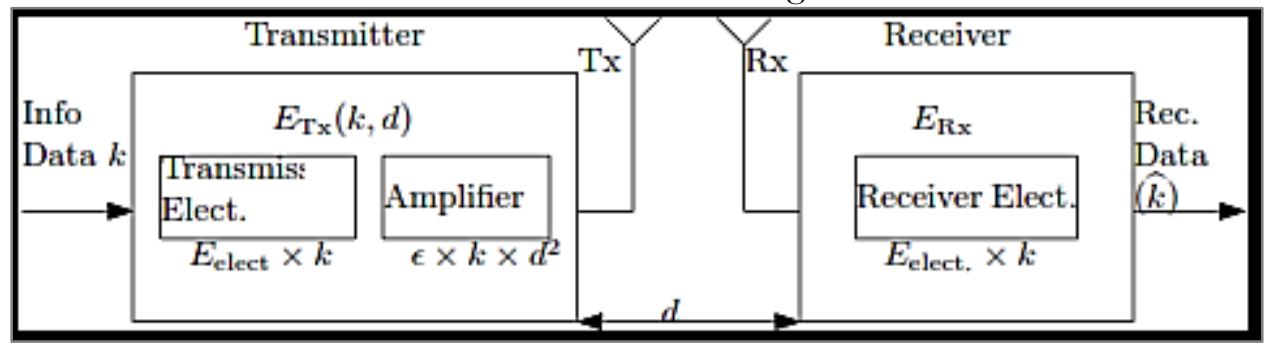

Figure 2. Block diagram of energy dissipation model.

\subsection{Mathematical model}

According to the radio model, $k$-bit message is transmitted covering a distance $d$ and the energy required to transmit the message is given by,

$$
\begin{array}{r}
E_{T x}(k, d)=k E_{\text {elect }}+\epsilon k d^{2} \quad \text { if } d<d_{o}(8) \\
=k E_{\text {elect }}+\epsilon k d^{4} \quad \text { if } d \geq d_{o}
\end{array}
$$

where, $E_{\text {elect }}$ represents the dissipated energy by the transmitter or the receiver circuit, $d$ denotes the distance between sender and receiver, $\epsilon$ is the energy dissipated by electronic and $d_{o}$ is the threshold distance. Noted that we consider the effect of free space and multi-path model in the dissipation model. Further to the dissipation model, free space path loss is considered for the transmission/reception between the $\mathrm{CNs}$ and $\mathrm{CH}$, whereas, multi-path model is for the $\mathrm{CH}$ to the BS. Then, the total energy $E_{t}$ consumed by the network for a round is given by $[5,6]$,

$\mathrm{E}_{\text {total }}=\left\{\sum_{\mathrm{i}=1}^{\mathrm{n}-1}\left(\mathrm{E}_{\text {elec }}+\mathrm{E}_{\mathrm{amp}} * \mathrm{~s}_{\mathrm{i}}^{2}\right)+\left(\mathrm{E}_{\text {elec }}+\mathrm{E}_{\mathrm{amp}} * \mathrm{~S}^{4}\right)\right\} * \mathrm{~m}$

where the average distance between the $\mathrm{SNs}$ and $\mathrm{CH}$ is $0.765 * \frac{M}{2}$ and $M$ is the dimension of sensing area [6].

\subsection{Numerical results}

We provide numerical results using the parameters given in Tab.1. To evaluate the performance of the proposed algorithm, large number of simulations were done for a given sensing area and the number of sensing nodes over the given parameters. MATLAB is used as a simulation tools to get results. 
Table 1. Simulation Result Parameters.

\begin{tabular}{|l|l|}
\hline Parameters & Values \\
\hline Sensing area & $100 \times 100$ \\
\hline SNs & 200 \\
\hline Initial E & $0.5 \mathrm{~J}$ \\
\hline Message size & $4 \mathrm{k} \mathrm{bits}$ \\
\hline $\mathrm{E}_{\text {elect }}$ & $50 \mathrm{~nJ} / \mathrm{bit}$ \\
\hline$\epsilon$ & $0.0015 \mathrm{pJ} / \mathrm{bit}$ \\
\hline $\mathrm{d}_{0}$ & $70 \mathrm{~m}$ \\
\hline Data aggregation cost & $5 \mathrm{~nJ} / \mathrm{bit}$ \\
\hline
\end{tabular}

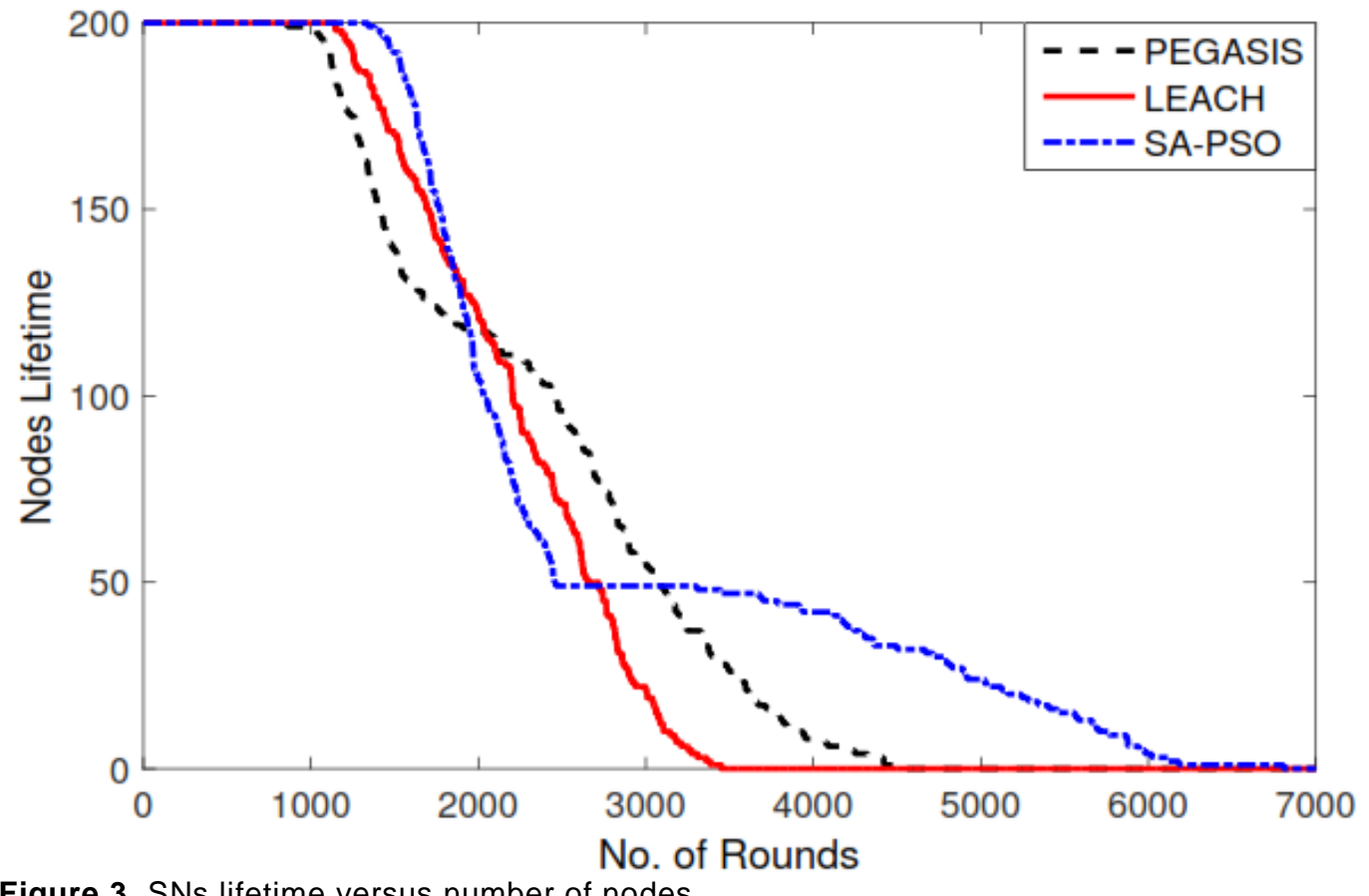

Figure 3. SNs lifetime versus number of nodes.

It is clearly seen in Fig. 3 that the lifetime of SNs in case of the proposed algorithm is more as compared to that of using LEACH and PEGASIS. It means that the performance of the network increases because of enhanced lifetime. From Fig. 3, it is seen that using SA-PSO, SNs die after completing more than 6000 rounds while for PEGASIS, nodes die out up to 4500 and for LEACH nodes die at 3500 . 


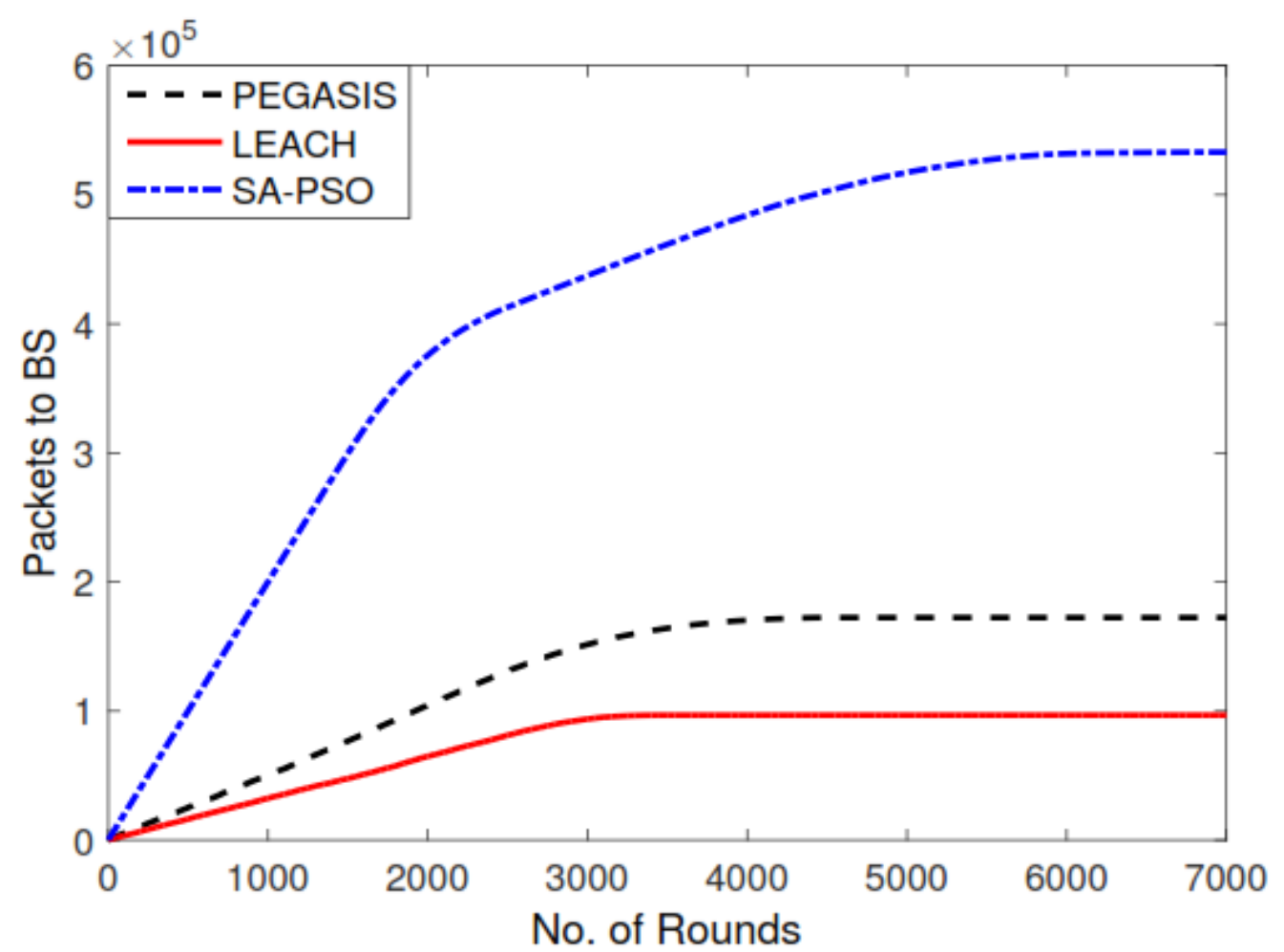

Figure 4. Data packet transmission to BS.

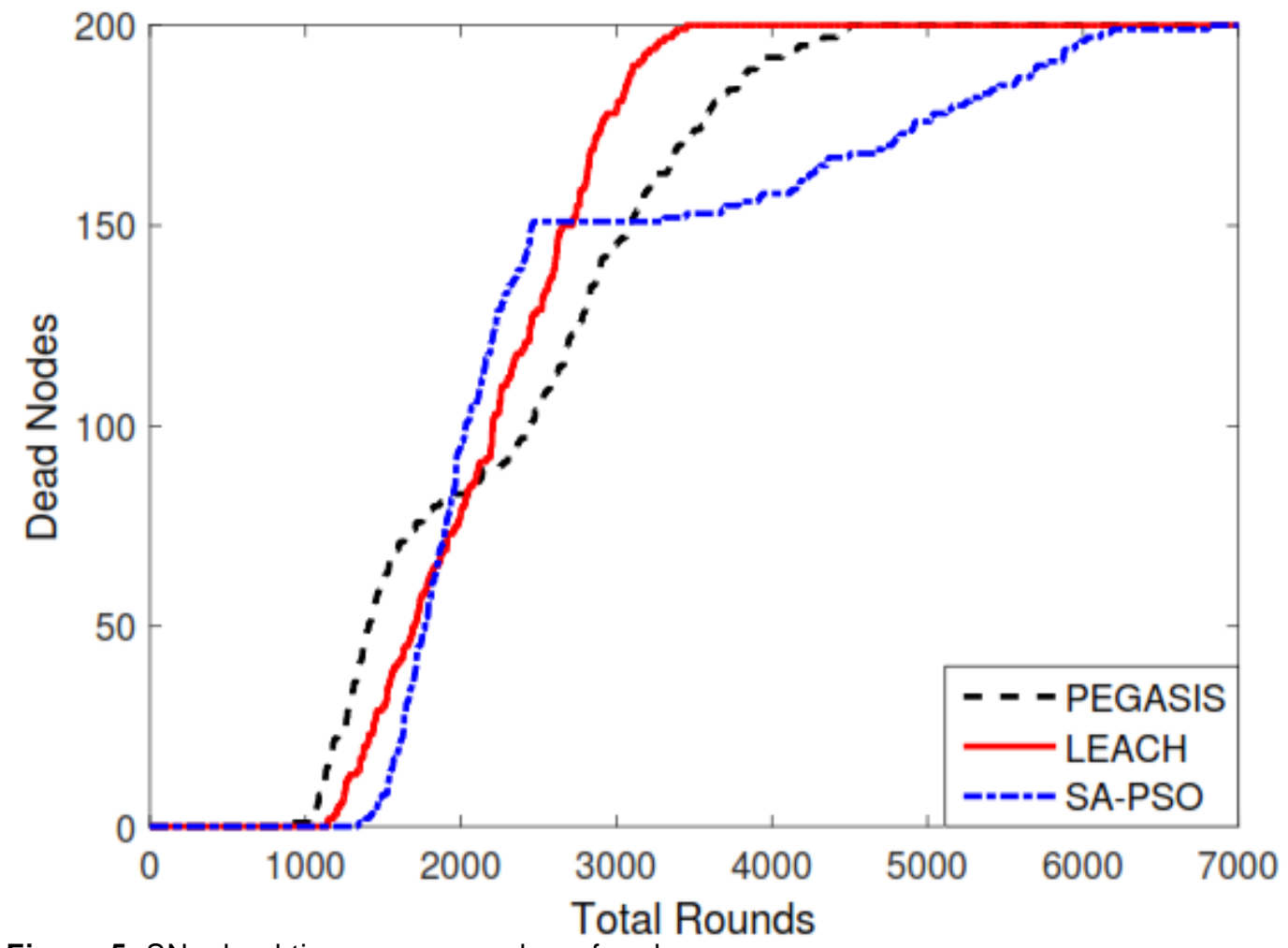

Figure 5. SNs dead time versus number of nodes.

Figure 4 presents the transmission of data packets to the BS. It is noted that SA-PSO outperform the other two LEACH and PEGASIS algorithms. It can be easily figured out that the data gathering efficiency is increased using the SA-PSO algorithm. Figure 5 shows dead nodes with the passage of time. It is 
seen that using SA-PSO, WSN has better performance because of longer lifetime.

For bigger WSNs, the low death rate of nodes not only reduces the network lifetime but its stability as well. After looking results, it is observed that the SA-PSO algorithm is comparatively more energy efficient algorithm for WSN rather than LEACH and PEGASIS in terms of lifetime and packet transmission as shown in Fig. 3 and Fig. 4.

\section{CONCLUSION}

In this research paper, an energy efficient scheme for data gathering in WSN is presented. A novel SA-PSO algorithm is proposed in which best chain formation procedure is adopted. The presented analysis shows that SA-PSO outperforms than PEGASIS and LEACH in terms of network lifetime, communication overhead and rate of SNs deaths. From simulation results, it is confirmed that SA-PSO outperforms LEACH and PEGASIS by eliminating the communication overhead in cluster formation and introduces low latency since it forms a chain among SNs. It is seen that SNs using SA-PSO remains active for over 1500 rounds than PEGASIS and 2500 rounds than LEACH.

\section{ACKNOWLEDGMENTS}

The authors would like to thank Dr. Ishtiaq Ahmad and Dr. Noor M. Sheikh for the follow discussion throughout the research work and thanks to Higher Education Commission Islamabad (HEC), Pakistan for their financial support to present the research work.

\section{REFERENCES}

S. Kalantary, and S. Taghipour, "A survey on architectures, protocols, applications, and management in wireless sensor networks", Journal of Advanced Computer Science and Technology, vol. 3, no. 1, (2014), pp. 1-11.

S. Gurbhej and K. Rajneet, "Review on Data dissemination and gathering in Wireless Sensor Networks", International Journal of emerging research in management and Technology, vol. 2013, no. 1, (2013), pp. 63--67.

S. A. Imam, V. K. Sachan, and V. P. Dubey, "Design and analysis of energy efficient communication methods for wireless sensor networks", Telecommunications and Radio Engineering, vol. 74, no. 19, (2015), pp. $1705-1714$.

Y. Wu, Z. Mao, S. Fahmy, and N. B. Shroff, "Constructing maximum-lifetime data-gathering forests in sensor networks", IEEE/ACM Transactions on Networking, vol. 18, no. 5, (2010), pp. 1571--1584.

C. Karakus, A. B. Gurbuz, and B. Tavli, "Analysis of energy efficiency of compressive sensing in wireless sensor networks", IEEE Sensors Journal, vol. 13, no. 5, (2013), pp. 1999--2008.

Yongming Qin, Qiuling Tang, Ye Liang, Xiuyu Yue, Xian Li, "An EnergyEfficient Cooperative MIMO Scheme for Wireless Sensor Networks", 14th IEEE International Conference on Computational Science and Engineering. Dalian, China, (2011), pp. 471--474.

X. He, X. Zhou, M. Juntti, and T. Matsumoto, "Data and error rate bounds for binary data gathering wireless sensor networks", Proceedings of the IEEE 
16th International Workshop on Signal Processing Advances in Wireless Communications (SPAWC '15). Stockholm, Sweden, June (2015), pp. 505-509.

M. Radi, B. Dezfouli, K. A. Bakar and M. Lee, "Multipath Routing in Wireless Sensor Networks: Survey and Research Challenges", Sensors, vol. 12, no. 1, (2012), pp. 650--685.

X. Xing, D. Xie, G. Wang, "Energy-balanced data gathering and aggregating in WSNs: a compressed sensing scheme", International Journal of Distributed Sensor Networks, vol. 10, no. 11, (2016), pp. 186.

K. Navdeep, S. Deepika, S. Prabhdeep, "Classification of Hierarchical Routing Protocols in Wireless Sensor Network: A Survey", International Journal of P2P Network Trends and Technology, vol. 3, no. 1, (2013), pp. 56--61.

M. Tong and M. Tang, "LEACH-B: An Improved LEACH Protocol for Wireless Sensor Network", 2010 6th International Conference on Wireless Communications Networking and Mobile Computing (WiCOM), Chengdu, China, Oct., (2010), pp. 1--4.

HU Sen-lai, ZHANG Yu, JIN Xin-yu, LI Hui-zhong, "Improvement of PEGASIS Algorithm in Wireless Sensor Networks Using GA", Journal of Jiangnan University, vol. 4, (2008), pp. 1-10.

J. Kennedy and R. C Eberhart, "Particle swarm optimization", Proc. IEEE International Conference on neural networks, IEEE service center, Piscataway, NJ, (1995), pp. 1942--1948.

O. Cakir, I. Kaya, A. Yazgan, O. Cakir, E. Tugcu, "Emitter Location Finding using Particle Swarm Optimization”, Radioengineering, vol. 23, no. 1, (2014), pp. 252--258.

Wen Liu, Zhiyuan Ma, Zhongliang Deng and Lianming Xu, "Energy Balance Algorithm based on LEACH", EEE 12th International Conference on Communication Technology, Nanjing, China, China, Nov., (2010), pp. 681 -684 .

N. Jasmine, J. J. Paulraj, and R. P. Prapoorna, "A Faster Routing Scheme for Stationary Wireless Sensor Networks - A Hybrid Approach”, International Journal of Ad Hoc, Sensor \& Ubiquitous Computing, vol. 1, no. 1, (2010), pp. 1--10.

M. T. Nguyen and K. A. Teague, "Tree-based energy-efficient data gathering in wireless sensor networks deploying compressive sensing”, Proceedings of the 23rd Wireless and Optical Communication Conference (WOCC'14). Newyark, NJ, USA, May (2014), pp. 1--6.

A. Norouzi, F.S. Babamir and A.H. Zaim, "A New Clustering Protocol for Wireless Sensor Networks using genetic algorithm approach", Wireless Sensor Networks, vol. 2011, no. 3, (2011), pp. 362--370.

M. Botta, M. Simek, "Adaptive Distance Estimation Based on RSSI in 802.15.4 Network”, Radioengineering Journal, vol. 22, no. 4, (2013), pp. 1162-1168.

S. K. Singh, P. Kumar and J. P. Singh, "A Survey on Successors of LEACH Protocol”, IEEE Access, vol. 5, (2017), pp. 4298--4328.

Mounir Arioua, Y. el Assari, Imad Ez-zazi and A. el Oualkadi, "Multi-hop Cluster Based Routing Approach for Wireless Sensor Networks", Procedia Computer Science, vol. 83, no. Supplement C, (2016), pp. 584--591.

N. G. Palan and B. V. Barbadekar and S. Patil, "Low energy adaptive clustering hierarchy (LEACH) protocol: A retrospective analysis", International 
Conference on Inventive Systems and Control (ICISC), Coimbatore, India, (2017), p. $\sim 1-12$.

Ez-Zazi, Imad, Arioua, Mounir, El Oualkadi, Ahmed and Lorenz, Pascal, "A Hybrid Adaptive Coding and Decoding Scheme for Multi-hop Wireless Sensor Networks", Wireless Personal Communication, vol. 94, no. 4, (2017), pp. 3017--3033.

M. N. Khan and M. Jamil, "Performance improvement in lifetime and throughput of LEACH protocol”, Indian Journal of Science and Technology, vol. 9, no. 21, (2016), pp.1-6.

Muhammad Nasir Khan, "Importance of Noise Models in FSO Communications," EURASIP Journal of Wireless Communication and Networking (EURASIP, JWCN), vol. 2014, no. 102, Dec. (2014), pp. 1-10.

Muhammad Nasir Khan and Mohsin Jamil, "Maximizing Throughput of Free Space Communication Systems using puncturing Technique,” in Arab. J. Sci. \& Eng. vol. 39, no.12, (2014), pp. 8925-8933.

Muhammad Nasir Khan and M. Jamil. "EXIT chart behavior for the Hybrid FSO/RF communication system" in 2015 10th International Conference on Information, Communications and Signal Processing (ICICS), Singapore, (2015), pp. 1-5. 